www.jmscr.igmpublication.org

Index Copernicus Value: 79.54

ISSN (e)-2347-176x ISSN (p) 2455-0450

crossref DOI: https://dx.doi.org/10.18535/jmscr/v7i6.108

\title{
Evaluation of Echocardiographic Profile in Chronic Kidney Disease in a Tertiary Centre Hospital
}

\author{
Authors \\ Dr Sumit ${ }^{1}$, Dr Mridul Chaturvedi ${ }^{2}$, Dr Kushal Pal Singh ${ }^{3}$ \\ ${ }^{1}$ Junior Resident P.G DEPT. of Medicine, S.N. Medical College Agra (INDIA) \\ ${ }^{2}$ Professor, P.G Dept. of Medicine, Agra (INDIA) \\ ${ }^{3}$ Junior Resident P.G DEPT. of Medicine, S.N. Medical College Agra (INDIA) \\ *Corresponding Author \\ Dr Mridul Chaturvedi \\ Professor P.G Dept. of Medicine, Agra (INDIA)
}

\begin{abstract}
Chronic kidney disease affects almost all systems of the body and results in various abnormalities, leaving significant morbidity and mortality. The prevalence of CKD progresses through stages and it is well known that patients are more likely to die than to progress to end stage renal disease. Heart and kidney are inextricably linked in terms of hemodynamic and regulatory functions. $2 \mathrm{D}$ echocardiography is a very helpful, inexpensive and non-invasive tool for detecting early cardiac dysfunction. In the present study cardiac profile of patients with chronic kidney disease with the help of echocardiography and to correlate the findings with the GFR.

Keywords: Echocardiography, chronic kidney disease, $L V H$.
\end{abstract}

\section{Introduction}

Patient with CKD have a lot of cardiac manifestation like development of obstructive CAD, pericardial effusion, pericardial calcification, patient with mildly reduced GFR (30-60 $\mathrm{ml} / \mathrm{min}$ ) are at increased risk of developing obstructive CAD. CAD contributes about $40-50 \%$ of death in patients who are receiving dialysis so recognition of cardiac manifestation is very important for early prevention of these death. The cardiomyopathy of the patient undergoing dialysis is mainly due to the presence of ischemic cardiomyopathy and morphofunctional alterations of the left ventricle (LV) in response to pressure and volume overload ${ }^{1,2}$. Early recognition of these cardiac abnormalities by echocardiography helps in better formulating of treatment plan and strategy of these patients.

Present study is to evaluate echo cardiographic profile in CKD Patient and its correlation with GFR as 2D Echocardiography is very helpful, inexpensive and noninvasive tool for detecting early cardiac dysfunction. Major abnormalities detected in these studies are $\mathrm{LVH}$, pericardial effusion mitral annular calcification, diastolic dysfunction and systolic dysfunction. In this study it is also reported that with increasing degree of CKD there is more prevalence of cardiac manifestations. 


\section{JMSCR Vol||07||Issue||06||Page 629-634||June}

\section{Material and Methods}

Present study was carried out in PG Department of medicine, those patients who have GFR less than $90 \mathrm{ml} / \mathrm{min} / 1.732 \mathrm{~m}^{2}$.were included in this study and patients with preexisting heart disease, Diabetes and history of nephrotoxic drugs, congenital heart disease, myocarditis were excluded from the study. In present study 100 patients were taken and creatinine clearance were calculated by the use of Cock Croft formula Cock Croftgault equation CRCL $=140$-Age $\mathrm{x}$ (wt in $\mathrm{kg}$ )for male/s creatinine $\mathrm{x} 72$

Cock Croftgault equation CRCL=140-AGE(wt in $\mathrm{kgs}$ )x 0.85 for female /s creatininex 7

After routine investigation following $\mathrm{ECHO}$ parameter were noted 1. Left ventricular end diastolic volume (LVEDV) 2.Left ventricular end systolic volume (LVESV) 3. Left ventricular ejection fraction (LVEF) 4.E/A ratio (for assessment of diastolic dysfunction) where $\mathrm{E}$ is early diastolic mitral inflow velocity and $\mathrm{A}$ is late diastolic mitral inflow velocity.

\section{Result}

In present study we have classified the patients according to GFR value.

Patients with GFR less than $90 \mathrm{ml} / \mathrm{min} / 1.732 \mathrm{~m}^{2}$ were included in study and patient with GFR less than $15 \mathrm{ml} / \mathrm{min} / 1.732 \mathrm{~m}^{2}$ labelled as end stage kidney disease (ESRD)

Table 1 distribution of cases according to serum creatinine value

\begin{tabular}{|c|c|c|c|c|c|}
\hline CKD Grading & Total & Mean & SD & F Value & P value \\
\hline 1 & 10 & 0.87 & 0.09 & 58.56 & $<0.0001$ \\
\hline 2 & 13 & 1.15 & 0.16 & 58.56 & $<0.0001$ \\
\hline 3 & 20 & 1.93 & 0.37 & 58.56 & $<0.0001$ \\
\hline 4 & 25 & 3.65 & 0.52 & 58.56 & $<0.0001$ \\
\hline 5 & 32 & 6.71 & 2.54 & 58.56 & $<0.0001$ \\
\hline & & & & & \\
\hline
\end{tabular}

Complete mean $+\mathrm{SD}=3.68 \pm 2.71$

In our study maximum patient that is 32 were in CKD grade 5 followed by 25 patient in CKD grade 4, 20 patients of CKD grade 3,13 patients of CKD grade 2, 10 patients of CKD grade 1. Complete Mean \pm SD $=3.68 \pm 2.72$, $p$ value $<0.0001$ as shown in table 1

Table 2 prevalence of LVH (Left ventricular hypertrophy)

\begin{tabular}{|l|c|c|c|c|c|c|}
\hline \multirow{2}{*}{$\begin{array}{l}\text { CKD } \\
\text { Grading }\end{array}$} & \multicolumn{2}{|c|}{ LVH PRESENT } & \multicolumn{2}{c|}{ LVH absent } & \multicolumn{2}{c|}{ Total } \\
\cline { 2 - 7 } & No. & $\%$ & No. & $\%$ & No. & $\%$ \\
\hline 1 & 3 & 30 & 7 & 70 & 10 & 100 \\
\hline 3 & 6 & 46.2 & 7 & 53.8 & 13 & 100 \\
\hline 4 & 11 & 55 & 9 & 45 & 20 & 100 \\
\hline 5 & 18 & 72 & 7 & 28 & 25 & 100 \\
\hline & 27 & 84.4 & 5 & 15.6 & 32 & 100 \\
\hline
\end{tabular}

As shown in table 2, the prevalence of LVH in echocardiography in our study 65 out of 100 patients.

The prevalence of $\mathrm{LVH}$ in echocardiography among CKD grade 1, grade2, grade3, grade4, grade5 were $30 \%, \quad 46.2,55 \%, 72 \%, \quad 84,4 \%$ respectively. The prevalence of LVH increased with increasing severity of renal impairment (30\% in CKD grade1 and $84.4 \%$ in CKD grade5). 
Table 3 prevalence of the pericardial effusion

\begin{tabular}{|l|c|c|c|c|c|c|}
\hline \multirow{2}{*}{$\begin{array}{l}\text { CKD } \\
\text { Grading }\end{array}$} & $\begin{array}{c}\text { Pericardial effusion } \\
\text { Present }\end{array}$ & & $\begin{array}{c}\text { Pericardial effusion } \\
\text { Absent }\end{array}$ & \multicolumn{2}{|c|}{ Total } \\
\cline { 2 - 7 } & NO. & $\%$ & No & $\%$ & No & $\%$ \\
\hline 1 & - & - & 10 & 100 & 10 & 100 \\
\hline 2 & - & - & 13 & 100 & 13 & 100 \\
\hline 3 & 2 & 10 & 18 & 90 & 20 & 100 \\
\hline 4 & 3 & 12 & 22 & 88 & 25 & 100 \\
\hline 5 & 10 & 31.5 & 22 & 68.75 & 32 & 100 \\
\hline
\end{tabular}

In table 3 the prevalence of pericardial effusion in echocardiography in our study 15 out of 100 patient.

The prevalence of pericardial effusion in echocardiography among chronic kidney disease

(CKD) grade 1, grade2,grade 3, grade4, grade5 were $0 \%, 0 \%, 10 \%, 12 \%, 31.25 \%$ respectively. The prevalence of pericardial effusion increased with increasing severity of renal impairment (10\% in CKD grade 3 and $31.25 \%$ in CKD grade5)

Table 4 Prevalence of the Mitral annular calcification

\begin{tabular}{|c|c|c|c|c|c|c|}
\hline \multirow{2}{*}{ Grading } & \multicolumn{2}{|c|}{$\begin{array}{c}\text { Mitral annular Calcification } \\
\text { Present }\end{array}$} & \multicolumn{2}{c|}{$\begin{array}{c}\text { Mitral annular calcification } \\
\text { Absent }\end{array}$} & \multicolumn{2}{c|}{ Total } \\
\cline { 2 - 7 } & No. & $\%$ & No. & $\%$ & No. & $\%$ \\
\hline 1 & - & - & 10 & 100 & 10 & 100 \\
\hline 2 & - & - & 13 & 100 & 13 & 100 \\
\hline 3 & 3 & 15 & 17 & 85 & 20 & 100 \\
\hline 4 & 12 & 48 & 13 & 52 & 25 & 100 \\
\hline 5 & 20 & 62.5 & 12 & 45.71 & 32 & 100 \\
\hline
\end{tabular}

As shown in table 4 the prevalence of Mitral annular calcification in echocardiography in our study 35 out of 100 patient. The prevalence of Mitral annular calcification in echocardiography among chronic kidney disease (CKD) grade1, grade2, grade3, grade4, grade5 were $0 \%, 0 \%$, $15 \%, 48 \%, 62.5 \%$ respectively. The prevalence of Mitral annular calcification increased with increasing severity of renal impairment $(15 \%$ in CKD grade 3 and $62.5 \%$ in CKD grade5).

Table 5.Prevalence of Systolic Dysfunction [LVEF (\%)]

\begin{tabular}{|l|c|c|c|c|c|}
\hline \multirow{2}{*}{$\begin{array}{l}\text { CKD } \\
\text { Grading }\end{array}$} & \multirow{2}{*}{ Total } & \multicolumn{2}{|c|}{ Normal } & \multicolumn{2}{c|}{ Abnormal } \\
\cline { 3 - 6 } & & No. & \% & No. & \% \\
\hline 1 & 10 & 9 & 90 & 1 & 10 \\
\hline 2 & 13 & 11 & 84.6 & 2 & 15.4 \\
\hline 3 & 20 & 16 & 80 & 4 & 20 \\
\hline 4 & 25 & 19 & 76 & 6 & 24 \\
\hline 5 & 32 & 9 & 28.12 & 10 & 31.25 \\
\hline
\end{tabular}

As shown in table 5 the prevalence of systolic dysfunction in echocardiography in our study $23 \%$ out of 100 patient.

The prevalence of systolic dysfunction in echocardiography among chronic kidney disease (CKD) grade 1, grade 2, grade 3 , grade 4 , grade 5,were $10 \%, 15.4 \%, 20 \%, \quad 24 \%, \quad 31.25 \%$ respectively. The prevalence of systolic dysfunction increased with increasing severity of renal impairment $(10 \%$ in CKD grade 1 and $31.25 \%$ in CKD grade 5). 
Table 6 Distribution of cases of According to E/A Ratio (Mean Value)

\begin{tabular}{|l|c|c|c|c|c|}
\hline $\begin{array}{l}\text { CKD } \\
\text { Grading }\end{array}$ & Total & Mean & SD & F Value & P Value \\
\hline 1 & 10 & 1.05 & 0.11 & & \\
\cline { 1 - 4 } 2 & 13 & 1.08 & 0.15 & \multirow{2}{*}{8.038} & $<0.0001$ \\
\cline { 1 - 4 } & 20 & 1.09 & 0.16 & & \\
\hline 4 & 25 & 1.36 & 0.50 & & \\
\hline 5 & 32 & 1.62 & 0.53 & & \\
\hline
\end{tabular}

Complete Mean $+\mathrm{SD}=1.32 \pm 0.46$

In our study on the basis of E/A Ratio(E is the early diastolic mitral inflow velocity and $\mathrm{A}$ is late diastolic mitral inflow velocity and $\mathrm{A}$ is late diastolic mitral inflow velocity ), 10 patients have mean value $=1.05 \pm 0.11$ were in CKD grade 1,13

CKD grade 2,20 patients have mean value $=1.09 \pm 0.16$ were in CKD grade 3,25 patients have mean value $=1.36 \pm 0.50$ were in $\mathrm{CKD}$ grade 4 , 32 patients have mean value $=1.62 \pm 0.53$ were in CKD grade5. (p value $<0.00001)$ patients have mean value $=1.08 \pm 0.15$ were in

Table 7 Distribution of cases according to Diastloic Dysfunction Grade (I to IV)

\begin{tabular}{|c|c|c|c|c|c|c|c|c|c|c|c|}
\hline \multirow{3}{*}{$\begin{array}{l}\text { Diastolic } \\
\text { dysfunction grading }\end{array}$} & \multicolumn{10}{|c|}{ CKD Grades } & \multirow{3}{*}{$\begin{array}{c}\begin{array}{c}\text { Total } \\
(\mathrm{N}=100)\end{array} \\
\text { No. }\end{array}$} \\
\hline & \multicolumn{2}{|c|}{$1(\mathrm{~N}=10)$} & \multicolumn{2}{|c|}{$2(\mathrm{~N}=13)$} & \multicolumn{2}{|c|}{$3(\mathrm{~N}=20)$} & \multicolumn{2}{|c|}{$4(\mathrm{~N}=25)$} & \multicolumn{2}{|c|}{$5(\mathrm{~N}=32)$} & \\
\hline & No. & $\%$ & No. & $\%$ & No. & $\%$ & No. & $\%$ & No. & $\%$ & \\
\hline NORMAL & 9 & 90 & 10 & 76.9 & 15 & 75 & 16 & 64 & 12 & 37.5 & 62 \\
\hline Abnormal & 1 & 10 & 3 & 23.1 & 5 & 25 & 9 & 36 & 20 & 62.5 & 38 \\
\hline $\mathrm{I}$ & 1 & 10 & 2 & 15.3 & 3 & 15 & 1 & 4 & 3 & 9.3 & 10 \\
\hline II & - & - & 1 & 7.7 & 2 & 10 & 3 & 12 & 5 & 15.6 & 11 \\
\hline III & - & - & - & - & - & - & 4 & 16 & 10 & 31.25 & 14 \\
\hline IV & - & - & - & - & - & - & 1 & 4 & 2 & 6.2 & 3 \\
\hline
\end{tabular}

As shown in table 7 the prevalence of diastolic dysfunction in CKD was $38 \%$ out of 100.The diastolic dysfunction in echocardiography among the CKD patients in our study showed a prevalence of diastolic dysfunction $10 \%$ in grade I CKD. Similarly the diastolic function in echocardiography among the CKD patients in our study showed a prevalence of diastolic dysfunction of $23.1 \%$ in grade 2 CKD. Similarly the diastolic function in echocardiography among the CKD patients in our study showed a prevalence of diastolic dysfunction of $25 \%$ in grade 3 CKD. Similarly the diastolic function in echocardiography among the CKD patients in our study showed a prevalence of diastolic dysfunction of $36 \%$ in grade 4 CKD Similarly the diastolic function in echocardiography among the CKD patients in our study showed a prevalence of diastolic dysfunction of $62.5 \%$ in grade $5 \mathrm{CKD}$

\section{Discussion}

The spectrum of CVD in CKD includes ischemic heart disease, congestive heart failure, arrhythmias

and peripheral vascular disease ${ }^{7}$ CVD kills patients with CKD more frequently and prematurely. Initially it was thought that this is limited to the ESRD population who were 20-30 times more likely to die of CVDs in comparison with the general population. This increase risk however is spread over the entire spectrum of CKD across all stages of CKD. The risk of CVD in CKD varies with the degree of renal impairment and proteinuria and depends on the rate at which these changes occur. The traditional risk factors for CVD such as increasing age, hypertension, dyslipidaemia, diabetes, smoking, and obesity are risk factors for CKD as well and hence are common in patients with CKD. The non-traditional or novel risk factors are uraemic specific or at least much more common in patients with CKD than in the general population. These include albuminuria, anemia, hyperparathyroidism, metabolic bone disease, hyperhomocysteinemia, malnutrition, apolipoprotein isoforms, inflammation, 
endothelial dysfunction dysfunction and oxidative stress. The various risk factors traditional and nontraditional tend to have an additive effect and hasten atherosclerosis and progression of $\mathrm{CKD}^{6}$.

The Doppler echocardiogram allows the evaluation of ventricular mass and volume and has an excellent accuracy for the detection of hypertrophy, in addition the Doppler derived techniques can generate information regarding ventricular relaxation and its dynamics of filling.

LV hypertrophy is highly prevalent in CKD and is associated with a clearly unfavourable prognosis; therefore it is a major target for intervention. The incidence of $\mathrm{LVH}$ increases with a progressive decline in renal function ${ }^{8}$. In our study The prevalence of LVH in echocardiography among CKD grade 1, grade2, grade3, grade4, grade5 were $30 \%, 46.2,55 \%, 72 \%, 84,4 \%$ respectively. The prevalence of $\mathrm{LVH}$ increased with increasing severity of renal impairment (30\% in CKD grade1 and $84.4 \%$ in CKD grade5).

In studies using different methodologies, the prevalence of systolic dysfunction of LV varies from 15 to $28 \%$ in patients of dialysis $(9,10,11)$.In our study the prevalence of systolic dysfunction in echocardiography among chronic kidney disease (CKD) grade 1, grade 2, grade 3, grade 4, grade 5,were $10 \%, 15.4 \%, 20 \%, 24 \%, 31.25 \%$ respectively. The prevalence of systolic dysfunction increased with increasing severity of renal impairment $(10 \%$ in CKD grade 1 and $31.25 \%$ in CKD grade 5).

Diastolic dysfunction is characterized by alterations in ventricular relaxation and compliance, frequently followed by a compensatory increase in filling pressure in more advanced stages. In our study the prevalence of diastolic dysfunction in CKD was $38 \%$ out of 100.The diastolic dysfunction in echocardiography among the CKD patients in our study showed a prevalence of diastolic dysfunction $10 \%$ in grade I CKD. Similarly the diastolic function in echocardiography among the CKD patients in our study showed a prevalence of diastolic dysfunction of $23.1 \%$ in grade 2 CKD.
Similarly the diastolic function in echocardiography among the CKD patients in our study showed a prevalence of diastolic dysfunction of $25 \%$ in grade 3 CKD. The diastolic function in echocardiography among the CKD patients in our study showed a prevalence of diastolic dysfunction of $36 \%$ in grade 4 CKD, the diastolic function in echocardiography among the CKD patients in our study showed a prevalence of diastolic dysfunction of $62.5 \%$ in grade 5 CKD.

In study conducted by Mukesh Laddha et al $(2014)^{3}$ echocardiography Pericardial effusion was noted in $14.3 \%$ of patients. Valvular calcification was noted in $7.1 \%$ of ESRD patients $^{[12]}$ D'Cruz IA et al $(1978)^{4}$ performed Echocardiography in 50 patients in chronicrenal failure. Pericardial effusions were detected in 33 and pericardial thickening in 22.In our study The prevalence of pericardial effusion in echocardiography among chronic kidney disease (CKD) grade 1, grade2, grade 3, grade4, grade5 were $0 \%, 0 \%, 10 \%, 12 \%, 31.25 \%$ respectively. The prevalence of pericardial effusion increased with increasing severity of renal impairment (10\%in CKD grade 3 and $31.25 \%$ in CKD grade5). Wang AY et al $(2003)^{5}$ Calcification complications are frequent among long-term dialysis patients. However, the prognostic implication of cardiac valve calcification in this population is not known. This study aimed to determine if cardiac valve calcification predicts mortality in long-term dialysis patients. Baseline echocardiography was performed in 192 on continuous ambulatory peritoneal dialysis to screen for calcification of the aortic valve, mitral valve or both. Valvular calcification was present in 62 patients. In conclusion, cardiac valve calcification is a powerful predictor for mortality and cardiovascular deaths in long-term dialysis patients.

\section{Conclusion}

India has become the capital for Diabetes with increasing prevalence of Diabetes more and more patients of CKD are seen in outdoor and indoor 
setting. Presently management of CKD needs multidisciplinary approach for which early recognition of cardiac complication in CKD is required, mechanism of cardiac complication in CKD is quite complex and multifactorial with the help of echocardiography early detection of these cardiac complication is possible which helps in better formulating the management strategy of CKD; present study is basically used for north Indian population. Unfortunately sample size is small so large data is needed before formulating any guideline in the management of cardiac complication of CKD.

\section{Bibliography}

1. McCullough PA: Why is chronic kidney disease the "spoiler" for cardiovascular outcomes?. J Am CollCardiol 2003; 41:725.

2. Stenvinkel P, Pecoits-Filho R, Lindholm B. Coronary artery disease in end-stage renal disease: no longer a simple plumbing problem. J Am SocNephrol. 2003; 14: 1927-39

3. Mukesh Laddha Vishal Sachdeva PM Diggikar, PK Satpathy, AL Kakrani ,Echocardiographic Assessment of Cardiac Dysfunction in Patients of End Stage Renal Disease on Haemodialysis, Journal of the association of physicians of India January 2014 Vol. 62

4. D'Cruz IA, Bhatt GR, Cohen HC, Glick G., Echocardiographic detection of cardiac involvement in patients with chronicrenal failure, Arch Intern Med. 1978 May;138(5):720-4.

5. Wang AY, Wang M, Woo J, Lam CW, Li PK, Lui SF, et al. Cardiac valve calcification as an important predictor for all-cause mortality and cardiovascular mortality in long-term peritoneal dialysis patients: a prospective study. J Am Soc Nephrol. 2003; 14: 159-68.
6. Gansevoort RT, Correa- Rotter R, Hemmelgarn BR et al. Chronic kidney disease and cardiovascular risk: epidemiology mechanisms and prevention. Lancet 2013;382:339-52.

7. Herzog CA, Asinger RW, Berger AK et al. Cardiovascular disease in chronic kidney disease. A clinical update from kidney Disease: improving Global Outcomes (KDIGO).Kidney int 2011;80:572-86.

8. Levin A, Singer J, Thrompson CR, Ross $\mathrm{H}$, Lewis M:Prevalant left ventricular hypertrophy in the predialysis population: Indentifying opportunities for intervention. Am J Kidney Dis 1996;27:3476-354.

9. McGregor E,Jardine AG,Murray LS, Dargie HJ, Rodger RS ,Junor BJ, et al:Preoperative echocardiographic abnormalities and adverse outcome following renal transplantation. Nephrol Dial Transplant 1998;13:1499-1505.

10. Parfrey PS, Foley RN, Harnett JD,Kent GM, Murray DC, Barre PE: Outcome and risk factors for left ventricular disorders in chronic uraemia. Nephrol Dial Transplant 1996;11:1277-1285

11. Barberato SH, Pecoits Filho R:Prognostic value of left atrial volume index in hemodialysispatients. Arq Bras Cardiol 2-7;88:650. 